\title{
Characterisation and calibration of a large aperture (1.6 m) ka-band indoor passive millimetre wave security screening imager
}

\author{
Neil A. Salmon*, Louise Kirkham, Peter N. Wilkinson \\ Jodrell Bank Centre for Astrophysics, University of Manchester, Manchester, M13 9PL, UK
}

\begin{abstract}
A large $1.6 \mathrm{~m}$ aperture mechanically scanning quasi-optical millimetre wave imaging system designed for active use ${ }^{[1]}$ has been investigated for its passive millimetre wave (PMMW) imaging capabilities. This raises new challenges for this imager, as the person / background contrast in indoor security scenarios at these frequencies is in the region of 6-7 K. The system is sensitive over $24-28 \mathrm{GHz}$ and has a radiometric sensitivity measured at $6.5 \mathrm{~K}$ in a single frame, at a frame rate of $8.8 \mathrm{~Hz}$. It was found that the focal plane receiver array radiated an excess noise ratio (ENR) of typically 9 $\mathrm{dB}$. This radiation re-entered the focal plane array receivers after reflection from the imager optical components typically with a standard deviation of $17 \mathrm{~K}$ over the mechanical scan associated with a single image frame. Reflected from subjects in the field of view, it was typically $400 \mathrm{~K}$ from a $100 \%$ reflective surface, and $40 \mathrm{~K}$ to $100 \mathrm{~K}$ from a human subject. This radiation, termed self-emission in this paper, is a feature of many PMMW imaging systems that use highgain electronic amplifiers in their receiving systems. The effectiveness in the use of a calibration mechanism to subtract self-emission reflected from the optical components and a large area quarter wave plate to minimising the amount of self-emission reflected back from subjects is demonstrated. The present system would benefit greatly from replacement amplifiers of larger bandwidth, lower self-emission and lower noise figure. It was also found that the millimetre wave emission from fluorescent room lighting entered the optics of the imager and modulated the received signals at $100 \mathrm{~Hz}$. The mean level of emission was measured at $10 \mathrm{~K}$ with a $\pm 10 \mathrm{~K}$ modulation at $100 \mathrm{~Hz}$ for per mode for a $100 \%$ reflecting surface in the room. The best solution to the problem of emission from fluorescent lights was to turn them off and use incandescent lighting.
\end{abstract}

Keywords: Quasi-optical, passive, millimetre wave, imager, MMIC, security screening, fluorescent, non-metallic, CFL

\section{INTRODUCTION}

The challenge of raising the contrast in millimetre wave imaging systems for security screening has previously been to artificially raise the radiation temperature of the illumination by using amplified electronic noise which was spatially decorrelated using mode scrambling cavities. However, the illumination from these mode scrambling cavities generates its own artefacts and these are unhelpful when trying to discriminate non-metallic threats. The work in this paper is to investigate purely passive imaging and to see how this system could be developed into one demonstrating competitive capabilities for portal security screening imaging.

\section{THE SYSTEM}

\subsection{System description}

The imager has a folded optical design with an aperture of $1.6 \mathrm{~m}$ with a 58 receiver linear focal plane array which is conically scanned using an off-axis spinning disk ${ }^{[1]}$ as illustrated in the schematic and photograph of Figure 1 . The sampling scheme in the focal plane is Rayleigh (beam-width) sampling in the plane parallel to the array and Nyquist (half beam-width) perpendicular to the array, achieved through the conical scan. The field of view of the system is $97 \mathrm{~cm}$ wide by $198 \mathrm{~cm}$ high at a range of $1.6 \mathrm{~m}$ from the front face of the imager and contains $\sim 17,400$ pixels. For passive use, this system is effectively sensitive to horizontal polarisation over the frequency band $24 \mathrm{GHz}$ to $28 \mathrm{GHz}$, although the system has sensitivity at lower levels all the way up to $40 \mathrm{GHz}$, which can be exploited for active imaging using sources

* Correspondence: Email: neil.salmon at Manchester.ac.uk; Telephone: +44 7921172892 
at these higher frequencies. The radio frequency (RF) amplifiers in the focal plane array employ three custom MMICs with some filtering to tailor the frequency response. The overall gains of the amplifiers and feed waveguide is around $\sim 58 \mathrm{~dB}$ and the responsivity of the detector is $\sim 400 \mathrm{~V} / \mathrm{W}$. The radiometric sensitivity of the system is measured at $6.5 \mathrm{~K}$ in a single frame; however this can be improved by longer periods of integration.
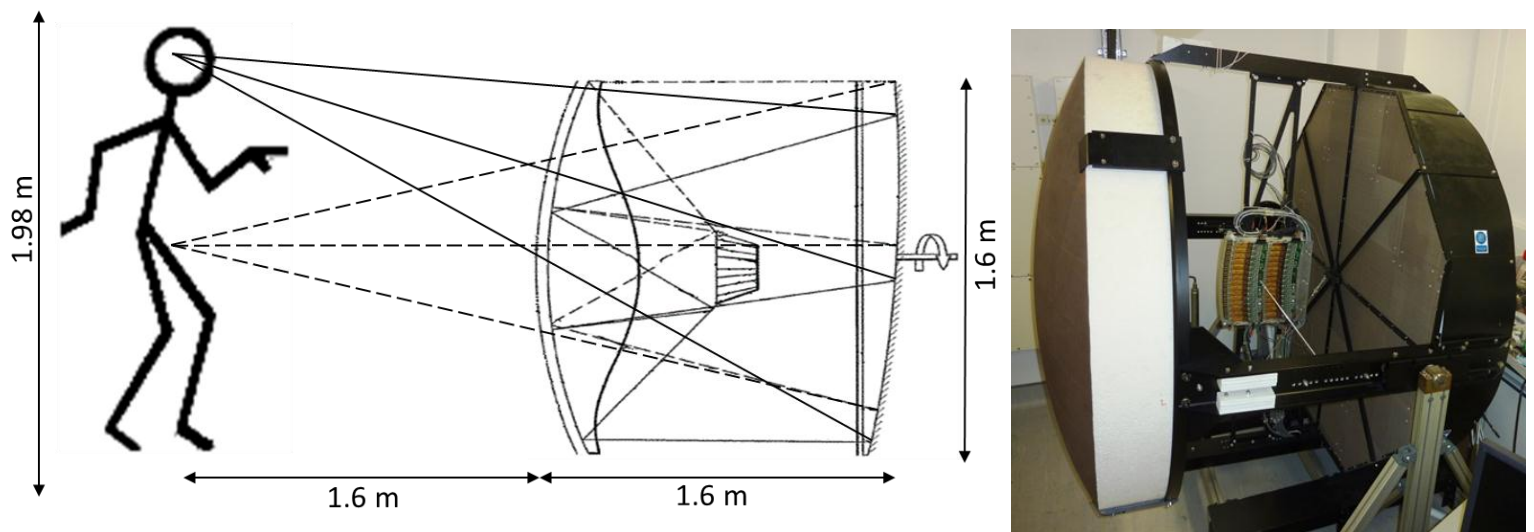

Figure 1: A schematic of the $1.6 \mathrm{~m}$ aperture ka-band passive imager illustrating corrector lens, focal plane array and off-axis scanning reflector and a photo of the system

\subsection{Measurement of depth of field}

The depth of field of the system was measured by moving a low energy Compact Fluorescent Light (CFL) bulb in the field of view $\pm 30 \mathrm{~cm}$ radially about the nominal subject range of $1.6 \mathrm{~m}$ during which time the system data acquisition was running. From these a series of images were created from which the $\sim 10 \mathrm{~dB}$ width of the measure lamp was estimated and this is plotted as a function of the range displacement in Figure 2. The lamp itself was oriented toward the imager with the smallest cross-section which is about $3.5 \mathrm{~cm}$ across, also shown in the figure. The graph in this figure indicates the width increases from $\sim 6 \mathrm{~cm}$ at the nominal $1.6 \mathrm{~m}$ range to around $18 \mathrm{~cm}$ at a $30 \mathrm{~cm}$ displacement from the centre of the field of view in both directions. The $3 \mathrm{~dB}$ beam-width of the point spread function at $1.6 \mathrm{~cm}$ is expected to be $\sim 2.8 \mathrm{~cm}$.

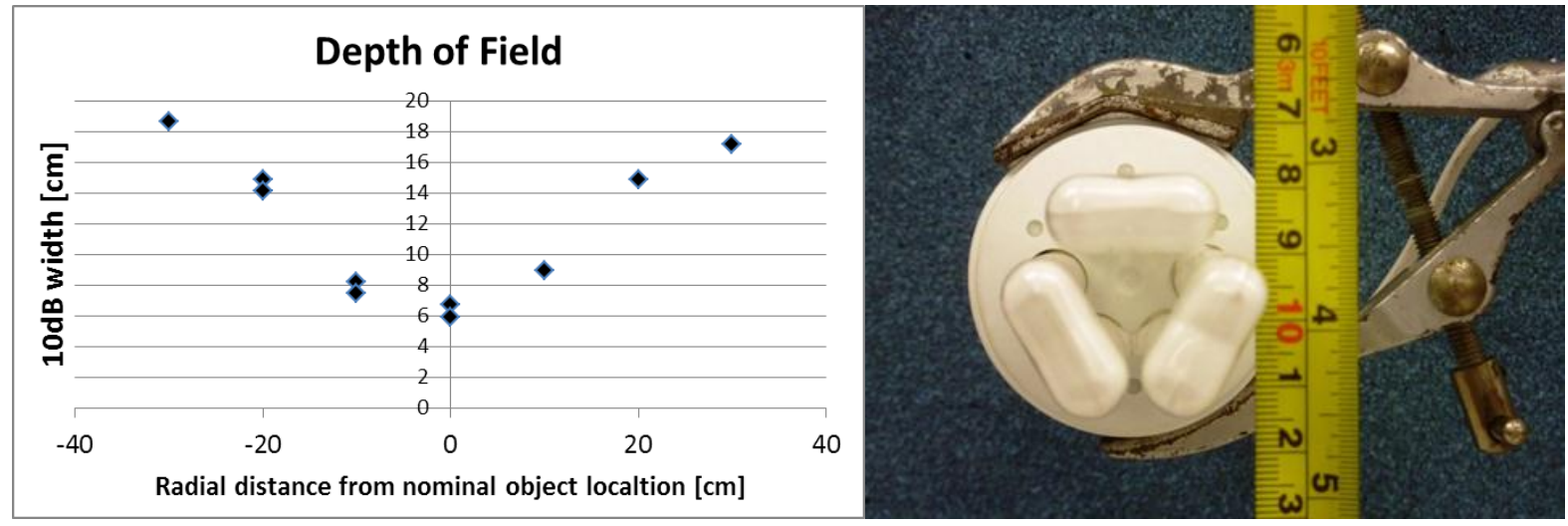

Figure 2: The depth of field (left) of the ka-band passive imager measured using a CFL bulb (right)

Commercially available low energy discharge CFL bulbs have been found to be a very convenient source of millimetre wave radiation for experimentation in PMMW imaging. In these experiments these lights have been found to have a radiation temperature of between $200 \mathrm{~K}$ and $500 \mathrm{~K}$ (dependent on lamp orientation) above ambient at $26 \mathrm{GHz}$. In many cases they are a convenient alternative to liquid nitrogen sources, particularly if the lamp can be calibrated absolutely against a liquid nitrogen standard. The lamp used in these experiments has a diameter of $3.5 \mathrm{~cm}$ and a length of $9 \mathrm{~cm}$.

\subsection{Noise temperature measurements}

The noise temperatures of the 58 channels in the focal plane array were measured using the y-factor test using liquid nitrogen and ambient temperature absorber as sources. The sources were placed directly in front of the feeds of the focal 
plane array. The mean of the noise temperatures and the standard deviation was measured at $1200 \pm 415 \mathrm{~K}$. There was an unusually large scatter in these noise temperatures with the highest being $2600 \mathrm{~K}$.

\subsection{RF amplifier spectral measurements}

The power spectrum output from the amplifiers was found to peak at $26 \mathrm{GHz}$ with a full $3 \mathrm{~dB}$ width of $4 \mathrm{GHz}$, although gain at lower level was present all the way up to $40 \mathrm{GHz}$.

\subsection{Radiometric sensitivity}

The radiometric sensitivity is given by the radiometer equation given in Eq. 1, where $T_{A N T}$ is antenna temperature, $T_{R E C}$ is the noise temperature of the system, $B_{R F}$ is the receiver radiation bandwidth and $t_{I N T}$ is the signal integration time. For this application it can be assumed that the antenna temperature is just the ambient temperature in the laboratory, as there is no external 'cold' sky radiation entering the laboratory. The $B_{R \mathrm{~F}}$ has been measured at $4 \mathrm{GHz}$. The integration time set by the low pass filters in the video electronics is $378 \mu \mathrm{s}$. With such an integration time, sampling at 300 points for a complete rotation of the scanning disk satisfies the Nyquist criteria for half beamwidth sampling in the direction of scan at the object location and sampling the low-pass filtered signal in the video electronics. The receiver noise temperature is given by Eq. 2 where $T_{0}$ is the ambient temperature of the imager, $L_{O P T I C S}$ is the loss in the optics from the feeds of the focal plane array to the beam at the subject location, measured at 5.55 using liquid nitrogen, and $T_{N}$ is the noise temperature at the feed location, measured to be $1200 \mathrm{~K}$. The precise origin of this loss in unknown, but the edge taper of the focal plane array feed on the focussing reflector was measured at $3 \mathrm{~dB}$ in the E-plane and $9 \mathrm{~dB}$ in the $\mathrm{H}$-plane. The quarter wave-plate has a measured transmission of $88 \%$ and two passes of this optical element are made in the system. Using this information the calculated sensitivity is $6.50 \mathrm{~K}$ in a single frame of the image.

$$
\Delta T=\frac{T_{A N T}+T_{R E C}}{\sqrt{B_{R F} t_{I N T}}}
$$

$$
T_{R E C}=T_{0}\left(L_{\text {OPTICS }}-1\right)+T_{N} L_{\text {OPTICS }}
$$

The radiometric sensitivity can also be measured experimentally by calculating the standard deviation on the absolutely calibrated temperature measurements of a contrastless scene, such as that of the absorber screen shown in Figure 9. The method used to measure the sensitivity is very accurate as it calculates the standard deviation on all of the receiver channels, then estimates the mean of this over receiver channels and a number of frames. This method it uses all of the data from all of the receiver channels over several minutes of integration, arriving at a figure of $6.54 \mathrm{~K}$ for a single

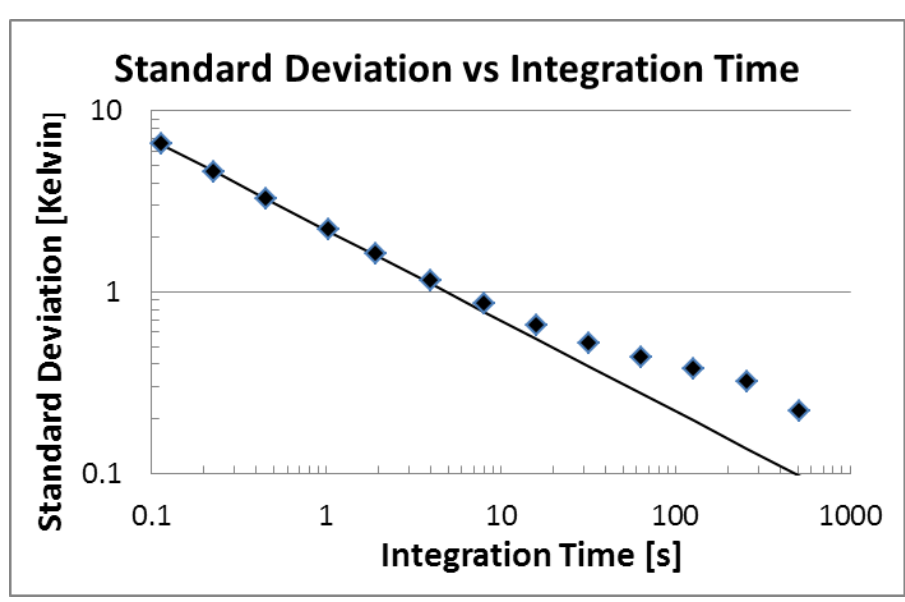

Figure 3: The measured standard deviation on the temperatures as a function of integration time. The solid line represents the root of time improvement in system sensitivity. For detection of non-metallic threats the sensitivity needs to be well below $1 \mathrm{~K}$. frame.

The benefit of recording the data digitally is that signal integration techniques can be used to improve the signal to noise ratio in the images. With the present system performance it would be necessary to use longer integration times to examine signatures of non-metallic security threats. The effectiveness of integration to reduce the noise can be examined by using digital low pass filters to integrate for periods longer than the inverse frame rate $(1 / 8.8 \mathrm{~Hz} \sim 114 \mathrm{~ms})$. A sequence of 10 minutes of data (5300 frames, a total of $184 \mathrm{MB}$ of data) was collected and calibrated. Radiation temperatures for each fixed point in space as a function of time were filter using integration times from $114 \mathrm{~ms}$ up to the full 10 minutes. This enabled the standard deviation on the temperature at each point in space to be estimated for this range of integration periods. The means of these standard deviations were then calculated over the total number of receiver channels and over the 300 sample points of one rotation of the scanner. The measured standard deviation as a function of integration period is shown in Figure 3. The solid line represents the square root of the integration time extrapolated from the single frame rate standard deviation of $6.54 \mathrm{~K}$. This shows the expected square root of time reduction in noise up to around $10 \mathrm{~s}$, after which the rate of 
improvement falls. After $10 \mathrm{~s}$ of integration the noise as dropped to $700 \mathrm{mK}$, which may be just sufficient to begin to see some non-metallic threats.

As all of the data is used in this technique to measure the standard deviation it is very accurate. Furthermore, as the standard deviation is calculated for temperatures at fixed points in space, the method is insensitive to the amount of selfemission in the system, the assumption being that this is constant with time at the fixed points in space.

\subsection{Calibration}

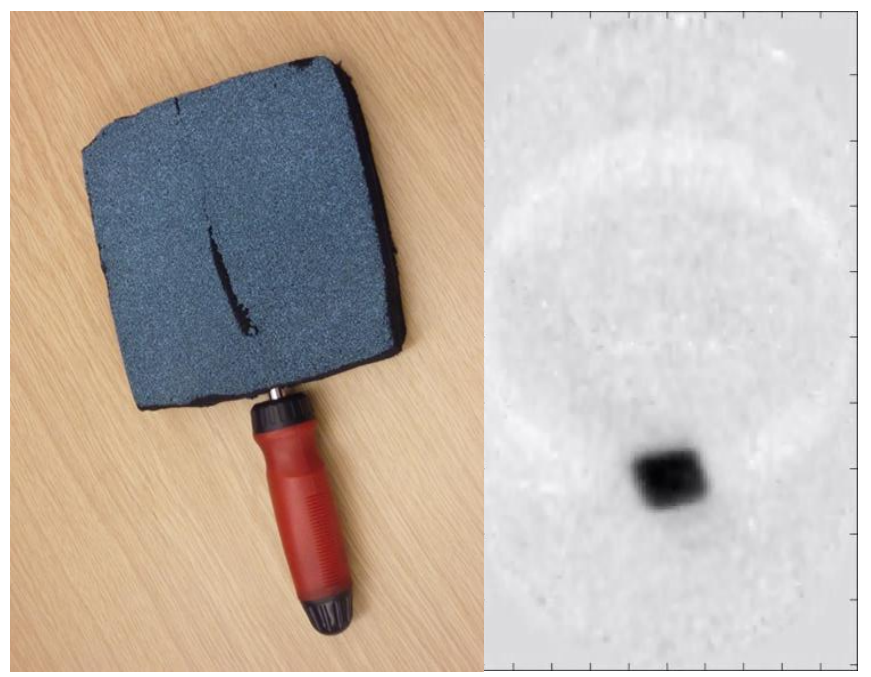

Figure 4: A $16 \mathrm{~cm}$ square section of absorber (left) is dipped in liquid nitrogen and physically moved from the bottom to top in the FOV at the nominal range of $1.6 \mathrm{~m}$ to absolutely calibrate all receiver channels, a snap shot image through this process is shown (right).
Generally the measured voltage from the detectors of the focal plane array vary with the channel index, $j$, and the rotation angle, $\theta$, of the scanning off-axis disk. Signals therefore need to be calibrated for gain and offset for each channel and each angle, which means effectively there is a calibration for every pixel, mathematically stated as in Eq. 3 .

$$
T(j, \theta)=V(j, \theta) \operatorname{gain}(j, \theta)+\operatorname{offset}(j, \theta)
$$

The variations in gain with $\theta$ arise due to the fact that the optical throughput in the system varies due to varying amounts of spillover and transmission of the optical elements. To first order the gain can be taken as constant over $\theta$ and a nominal value used taken from liquid nitrogen and ambient temperature source calibrations. In this calibration scheme a piece of absorber at liquid nitrogen temperature is moved in the field of view so it can be measured by all receiver channels, as illustrated in Figure 4. When done indoors against an absorber screen background, all other emission in the field of view has the ambient radiation temperature. This process calibrates the receiver absolutely. Combining this sensitivity measurement at the subject location with the noise temperature measured at the focal plane array feed established the overall loss in the optics to be a factor of $\sim 5.55$.

The varying amount of offset is due to the fact that there may be objects with different radiation temperatures in the beam spillover and the varying contributions of self-emission. The variation in offset with angle can be measured by viewing a scene with no contrast, such as a large area screen of millimetre wave absorber.

As a final clean-up of the image it is possible to perform a mean scene referencing, in which it is assumed that the mean temperature over a complete $360^{\circ}$ of scan is the same for all channels. This enables relatively good quality images to be achieved. Extensions to this method are possible based on assumptions that different receiver channels measuring the same part of the field of view, should measure the same radiation temperatures.

\subsection{Expected image contrast}

For an indoor imaging scenario with an absorber background the temperature contrast, $T_{C O N T}$, of the human body against this background is given by Eq. 4, where $T_{B D}$ and $R_{B D}$ is the physical surface temperature and reflectance of the human body and $T_{B G}$ is the ambient temperature in the room in Kelvin. Rearranging Eq. 4 gives the simpler expression Eq. 5 for the contrast. The reflectivity of the human body in this frequency band is in the range $45-55 \%$ for angles of incidence of $0^{\circ}$ to $45^{\circ}{ }^{[2]}$. For a human body with a surface temperature

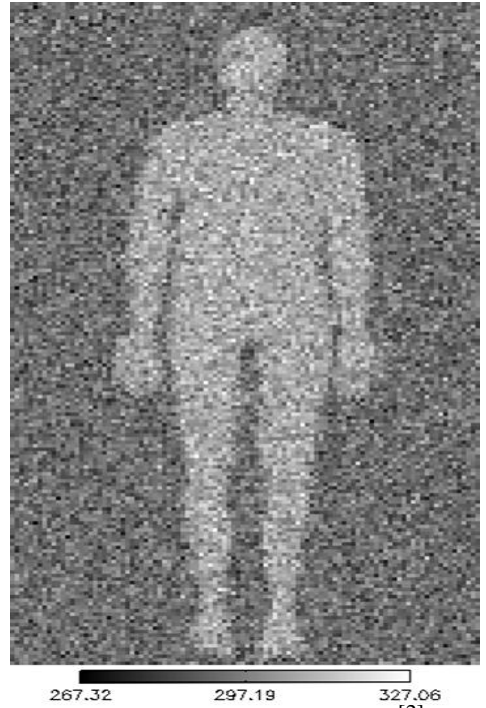

Figure 5: Scene simulation ${ }^{\lfloor 2\rfloor}$ of a person against an absorber background indoors when the system sensitivity is close to the subject background contrast. 
of $35^{\circ} \mathrm{C}$ and an ambient temperature of $21^{\circ} \mathrm{C}$ the contrast of the body against the background is in the region $6.3 \mathrm{~K}$ to 7.7 $\mathrm{K}$. This is a small contrast and given that the noise in a single frame is of this order, a real-time demonstration of security screening capabilities with this system in its current form may be challenging. A scene simulation ${ }^{[2]}$ of this scenario illustrates the type of imagery that would result in Figure 5.

$$
T_{C O N T}=T_{B D}\left(1-R_{B D}\right)+T_{B G} R_{B D}-T_{B G}
$$

$$
T_{C O N T}=\left(T_{B D}-T_{B G}\right)\left(1-R_{B D}\right)
$$

\subsection{Characterisation of the self-emission}

\section{SELF-EMISSION}

If the imager is viewing the emission from the absorber screen, as illustrated in Figure 9, a contrastless image should result. However, this is not the case, and an image with structure is measured as illustrated in Figure 6 . It is believed this structure is due to emission, referred to here as self-emission, emanating from the feeds of the focal plane array. This emission is reflected from the optical components of the system back into the feeds. If the emission measured on two adjacent channels of the imager is plotted as a function of the $360^{\circ}$ of rotation of scanner, and signal averaged for a few tens of seconds, the traces in Figure 6 result. This trace represents how the self-emission varies over the rotation angle of the scanner. There are some similarities between the two channels. The mean over the receiver channels and frames of the standard deviation over the $360^{\circ}$ rotation period is around $17 \mathrm{~K}$. The level of this emission can be measured accurately in a so-called background measurement and then subtracted from data as a calibration offset.
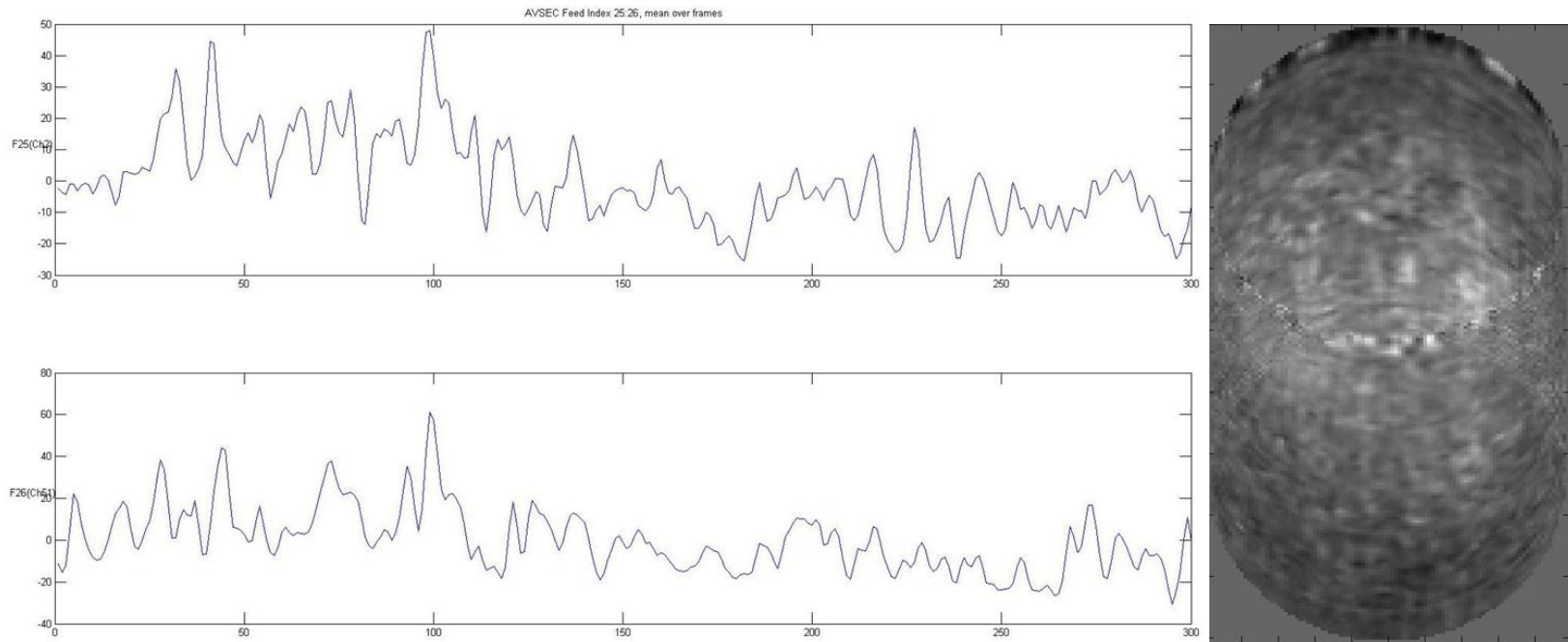

Figure 6: The image of self-emission (right) from internal reflections from the imager optics is shown as an image. The self-emission averaged on two adjacent channels (left) over a complete rotation of the scanner shows a degree of similarity.

\subsection{Origin of self-emission}

Self-emission is believed to be generated in the MMIC receiver package. The overall gain of the receiver from the focal plane array feeds to the input of the detector is approximately $58 \mathrm{~dB}$; this gain is achieved through three MMIC amplifiers. With such a high gain, a certain proportion of radiation is reflected within the package and then travels backwards through the system and out through the waveguide input feed. If there is sufficient radiation reflected back into the feed, it may also cause the amplifiers to oscillate. This level of emission from the feed is high, measured here to be equivalent to an excess noise ratio of typically $9 \mathrm{~dB}$ (but occasionally as high as $17 \mathrm{~dB}$ ) at the waveguide feed. Particularly for portal type security applications, minimisation of self-emission is critical, as in a well-designed low loss optical system, the proportion of this radiation reflected back from the subject into the imager can be large. This gives rise to glint effects which generate false alarms in security screening algorithms. 


\subsection{Effect of self-emission on imagery}

The self-emission which exits from the objective of the imager can be reflected off subjects and appears as glint in the imagery. Experiments were conducted to measure the magnitude of this effect. To measure the level of emission, experiments were conducted in which a large rectangular plane metal reflector was moved around in the beam approximately normal to the imager view direction. At positions where the normal of the plane surface matched exactly the view direction a perfect retro-reflection could be seen and a large spike appeared on the receiver outputs, typically around the level of $400 \mathrm{~K}$ but occasionally at $2500 \mathrm{~K}$ above ambient, as illustrated in Figure 7 . Given the 5.55 loss in the optics this is equivalent to an excess noise ratio of typically $9 \mathrm{~dB}$ to $17 \mathrm{~dB}$ on occasions.

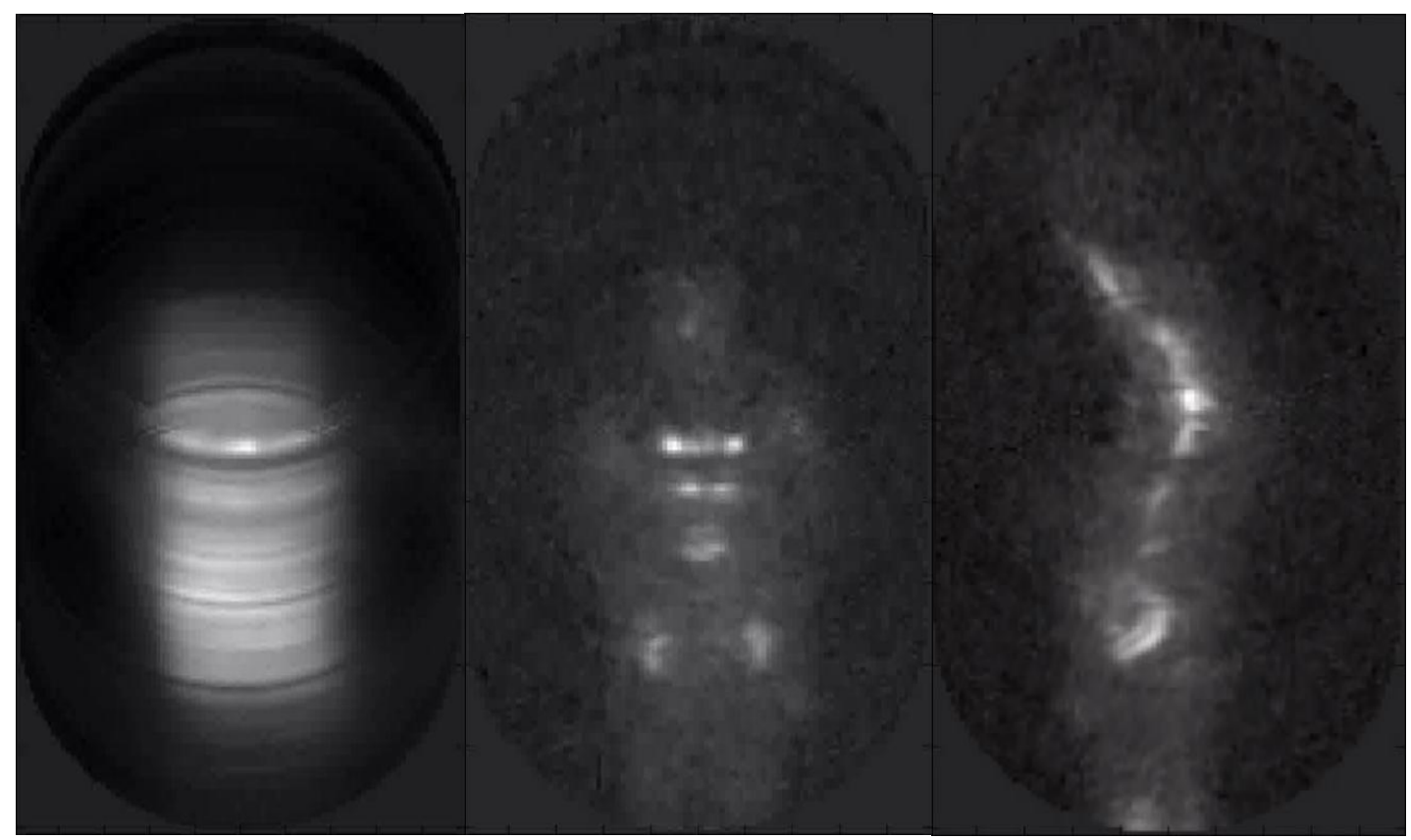

Figure 7: Self-emission reflected back from a plane rectangular metal reflector (left) appears as glint with radiation temperatures typically $400 \mathrm{~K}$ above the background. As a human subject (centre - front-on, right side-on) also reflects specularly, body surfaces normal to the view direction show glint having radiation temperatures $\sim 40 \mathrm{~K}-100 \mathrm{~K}$ above the background.

Self-emission reflected back from a human subject was observed to have the largest signatures when reflected back from those body surfaces normal to the imager view direction. This can be seen in Figure 7, where a person viewed front-on has bright spots on regions of the chest and the hips. Also illustrated in the figure are the bright spots from a person viewed side on with arms raised. Glint reflection is seen from the arms, down the side of the person to the hips. This glint type reflection is explained by the fact that the human body is a specular reflector at these wavelengths. For security imaging this phenomenon is a characteristic of spatially coherent illumination and needs to be eliminated to give the system a better performance.

\subsection{Self-emission - mitigation by quarter wave plate}

The self-emission radiated by the imager towards subjects must be horizontally polarised to escape from the front of the optical system. Glint effects can therefore be reduced by placing a quarter wave-plate directly in front of the imager, as shown in Figure 8. This converts the horizontally polarised self-emission to right-hand circularly polarised radiation, which when reflected back from the subject, becomes left-hand circularly polarised. When this travels back through the quarter wave plate it becomes vertically polarised and gets reflected out of the beam by the curved polarising grid on the front of the imager, and so does not enter the imaging optics. The quarter wave plate is made from meanderline, is similar to that used as an optical component in the imager, and has had its transmission measured at $88 \%$.

EMISSION FROM FLUORESCENT LIGHTS 

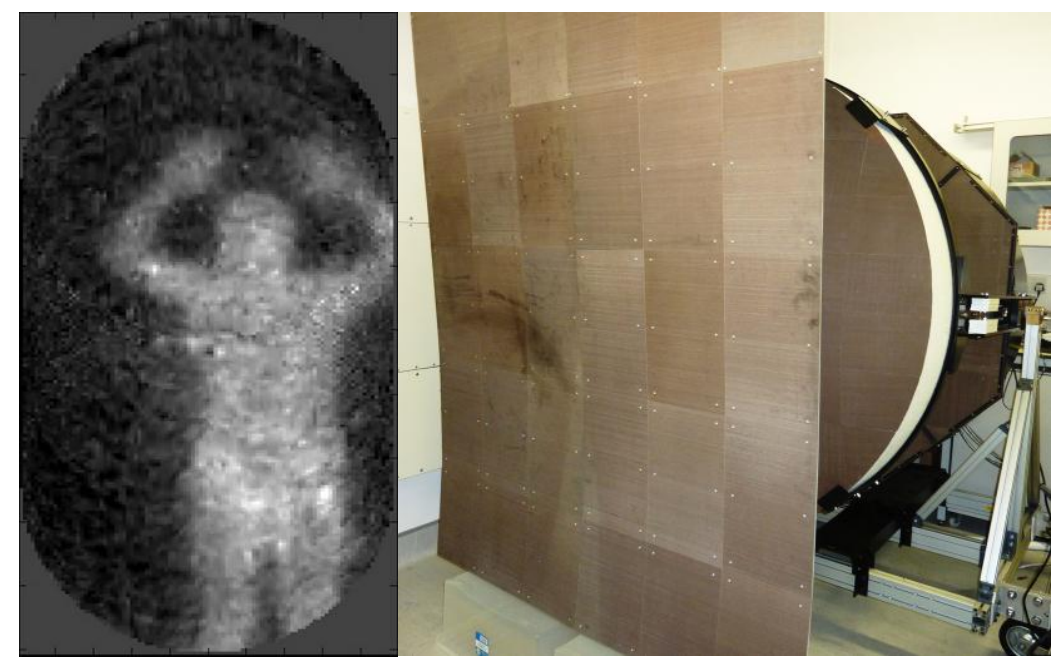

Figure 8: The glint effects of self-emission reflected back from a subject are minimised as illustrated in the image (left) by the use of a quarter wave-plate directly in front of the imager (right). To generate this image

$29 \mathrm{~s}$ of signal integration were used.
lights in the laboratory where turned on and off four times. The resulting time trace on one of the channels is also shown in Figure 9. During the on period the mean level of the radiation temperature is increased by $5 \mathrm{~K}$ whilst there is a modulation at $\pm 5 \mathrm{~K}$. As the level of emission is greater for receiver channels closer to the bottom of the focal plane array, it is believed that emission enters the system through the beam-spillover.
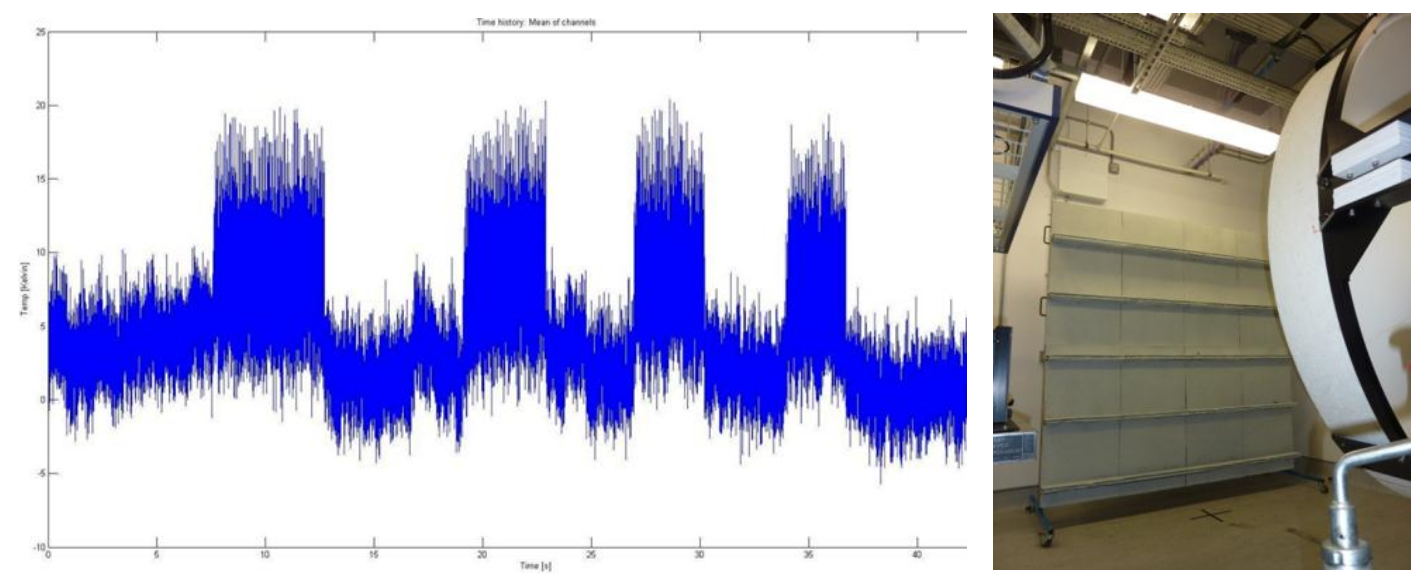

Figure 9: Millimetre wave emission from fluorescent lights being switched on/off four times over a short period appears as a rise in the radiation temperature of $\sim 5 \mathrm{~K}$ with a $\pm 5 \mathrm{~K}$ modulation. Viewing the absorber screen should generate a contrastless scene, but the fluorescent lamp emission enters the imager via beam spillover.

When the time trace of the signal from the above experiment is examined more closely it can be seen that there is an almost sinusoidal variation $( \pm 5 \mathrm{~K})$ in the signal with a period of $10 \mathrm{~ms}$ as can be seen in Figure 10 . Plotting the spectrum of this signal shows a raised spectral content of $3.4 \mathrm{~K}$ centred on $100 \mathrm{~Hz}$ which is also shown in the figure. This spectral content vanishes when absorber material is placed in front of the feeds. The effect of this emission on the image is to generate a banding structure, as shown in Figure 10.

\subsection{Fluorescent light emission measured when imaging the $100 \%$ reflecting metal foil}

With the imager turned to face a screen of $100 \%$ reflective metal foil, as illustrated in Figure 11, the level of the millimetre wave emission from the lights is raised by a factor of two. This can be seen in the time trace of the figure 
whereby the modulation has increase to a level of $\pm 10 \mathrm{~K}$, the spectral content rising to $6 \mathrm{~K}$ centred on $100 \mathrm{~Hz}$. The time trace of this modulation is much less like a sinusoid, believed to be so due to the increased level of self-emission entering the system, even though the larger part of this should have been subtracted as an offset in the background calibration.
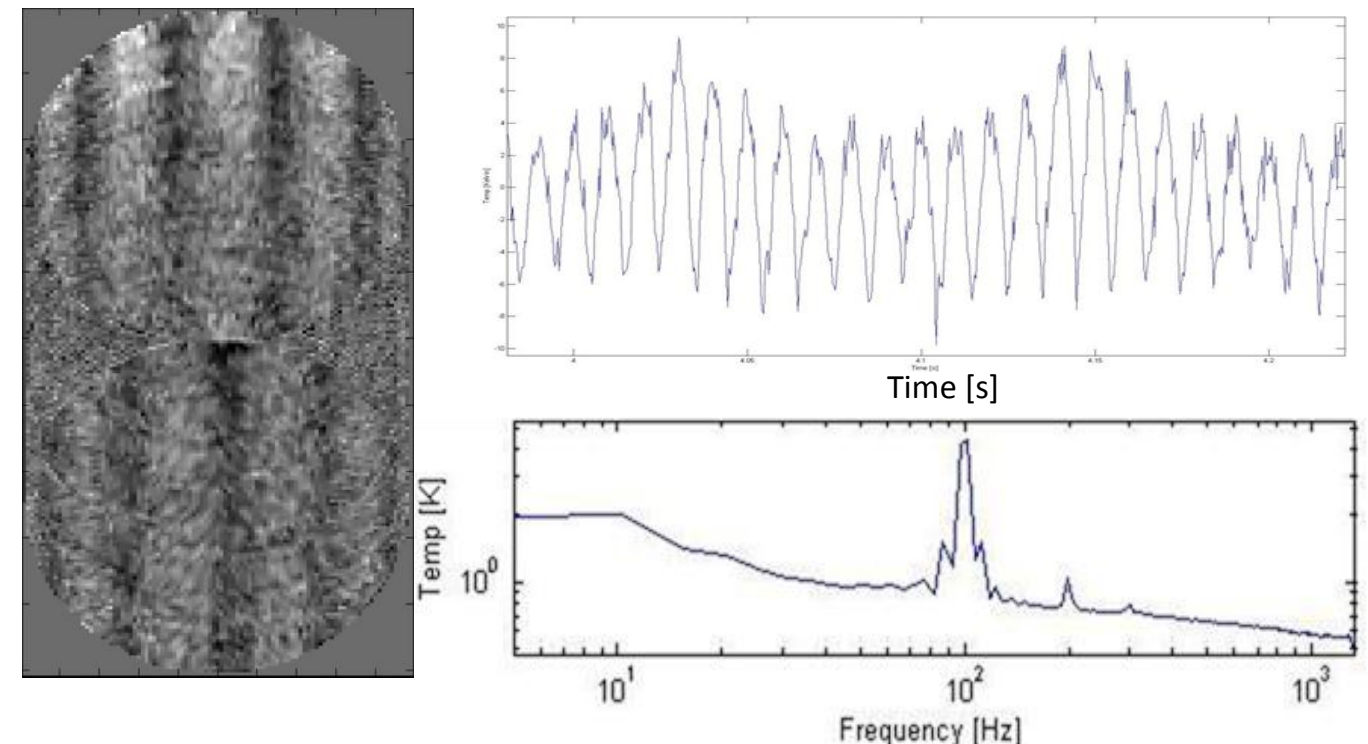

Figure 10: The modulation of millimetre wave emission $( \pm 5 \mathrm{~K})$ from fluorescent lights appears at a frequency of $100 \mathrm{~Hz}$, as is apparent in the time trace and the spectrum, even when in this case the absorber screen is viewed. The effect in the image is to generate a banding structure, as can be seen in the figure.

If the fluorescent lights in the room were turned off or absorber covers the feeds or the focal plane array, a large amount of the spectral content at $100 \mathrm{~Hz}$ vanishes. With the lights turned off the signal spectrum measured is that shown in Figure 12. The spectral content centred on $100 \mathrm{~Hz}, 200 \mathrm{~Hz}$ and $300 \mathrm{~Hz}$ is measured at $0.85 \mathrm{~K}, 0.87 \mathrm{~K}$ and $0.76 \mathrm{~K}$ respectively. Although the level has dropped considerably, these residual levels are probably generated through nonlinear effects in electronic components being excited by $50 \mathrm{~Hz}$ mains. This may be generated by power supply rectification of the mains and probably gets on to signals through DC power lines or through inductive coupling. The measurement technique in the estimation of the spectral signal content is very powerful as all of the spectra from all channels and all frames over a data acquisition time of tens of seconds are averaged to produce a single spectrum.

It is noted in these experiments that the millimetre wave emission from the lights vanishes immediately the lights are either turned off or the exciting voltage on the tube falls to zero. This supports the argument that the emission is generated by bremsstrahlung and that after excitation electron energy is lost relatively quickly as the collision frequencies of electrons in the plasma of fluorescent tubes is expect to be of the order or in excess of $\mathrm{GHz}$.

It is to be concluded from these measurements that the level of millimetre wave emission from fluorescent lighting in these rooms has a mean level of $\sim 10 \mathrm{~K}$ with $\pm 10 \mathrm{~K}$ modulation at $100 \mathrm{~Hz}$ when viewing a perfectly reflecting surface. This amount of radiation would enter per mode of any millimetre wave imaging system. For this particular system, it may be possible to reduce the level entering the imager by enclosing the optics with absorber. However, a much better solution would be to operate the system under incandescent lighting. There was no discernible level of millimetre wave emission from incandescent lamps, even when they were shone directly into the imaging system. 


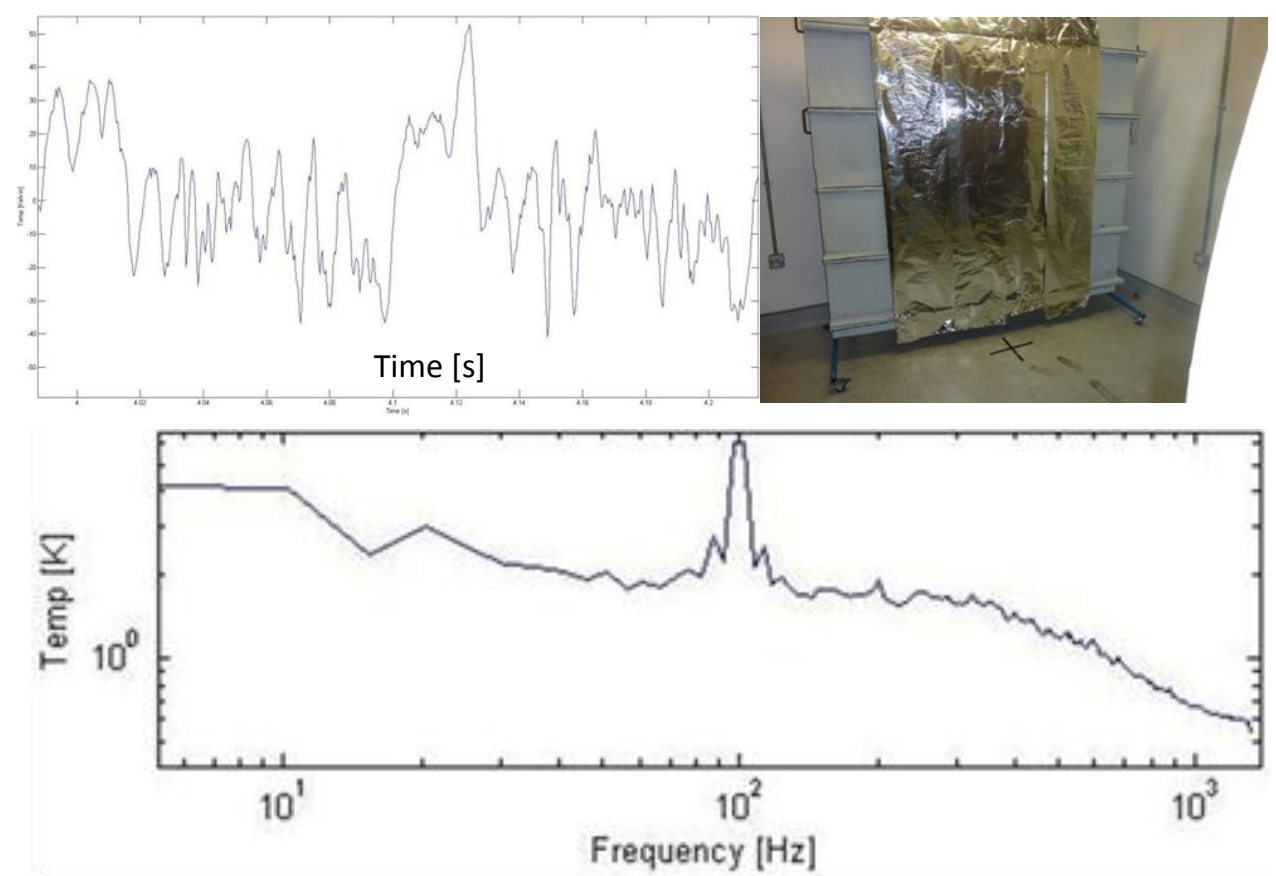

Figure 11: When the imager views the reflective screen, the level of millimetre wave emission modulated at $100 \mathrm{~Hz}$ from fluorescent lights effectively doubles to $\pm 10 \mathrm{~K}$, as can be seen in the signal time trace and spectrum.

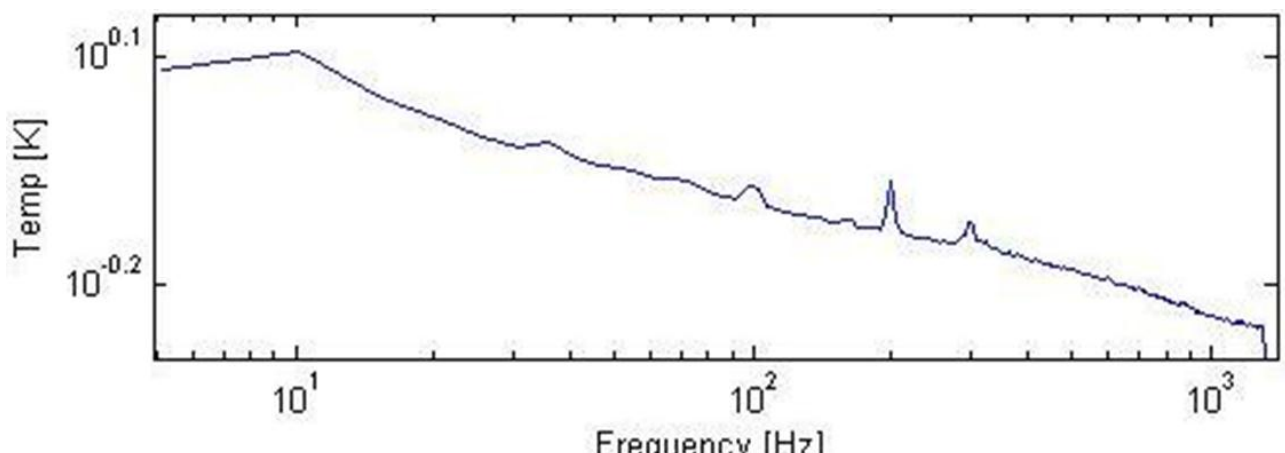

Figure 12: The signal spectrum measured during a period when all fluorescent lighting in experiment room was turned off 


\section{CONCLUSIONS}

A large aperture $1.6 \mathrm{~m}$ ka-band passive imaging system has been investigated and characterised for indoor portal security screening. The radiation bandwidth was measured at $24-28 \mathrm{GHz}$, the system sensitivity in a single frame at $6.5 \mathrm{~K}$ and the receiver noise temperature (at the waveguide feeds of the focal plane array) at $1200 \mathrm{~K}$. The system was absolutely calibrated using liquid nitrogen and then used to characterise self-emission (radiation emitted from the inputs of the receivers) and millimetre wave radiation from fluorescent lights. Reflected from the internal optics the self-emission had a standard deviation of $17 \mathrm{~K}$ over a rotation period of the mechanical scan. Reflected from a $100 \%$ metal reflector at the subject location the self-emission was typically $400 \mathrm{~K}$ above background. Reflected from a human subject it was $40 \mathrm{~K}$ to $100 \mathrm{~K}$ above background. These measurements are consistent with the radiation at the focal plane feeds having an excess noise ratio of typically $9 \mathrm{~dB}$. Emission from fluorescent room lighting was found to have a mean value of $10 \mathrm{~K}$ in the room and be modulated by $\pm 10 \mathrm{~K}$ at $100 \mathrm{~Hz}$, whereas the radiation temperature measured when looking directly at a low energy Compact Fluorescent Light (CFL) bulb filling the beam pattern was found to be at the level of $200 \mathrm{~K}$ to $500 \mathrm{~K}$ and dependent on orientation.

\section{FUTURE WORK}

To make better use of the system for the demonstration and study of the phenomena in portal security screening of nonmetallic threats, the radio frequency amplifiers should be replaced by improved units. Using amplifiers with a bandwidth increased to $26-40 \mathrm{GHz}$ and with a noise figure of $3 \mathrm{~dB}$ would lower the noise in the system by a factor of 4.5 . This would reduce the integration time necessary to get a $200 \mathrm{mK}$ sensitivity to around $5 \mathrm{~s}$, which would make the demonstration of a non-metallic screening system viable. In conjunction amplifiers with reduced self-emission should be used to reduce glint effects from subjects, which otherwise lead to false alarms. Examination of the contributions to the loss of x5.55 in the optics may lead to further improvements in the radiometric sensitivity. The performance which this demonstration system could deliver can be more compactly realised using an aperture synthesis imaging system, as the planar geometry is compatible with deployment in portal screening scenarios ${ }^{[3]}$.

\section{ACKNOWLEDGEMENTS}

The authors are greatly indebted to Professor Richard Winpenny and Med Benyezzar for providing laboratory space, the loan of electronic test gear and helpful advice in the area of health and safety in the laboratory.

\section{REFERENCES}

[1] Appleby, R “Whole body 35 GHz security scanner”, Radar Sensor Technology VIII and Passive MillimeterWave Imaging Technology VII, edited by Robert Trebits, James L. Kurtz, Roger Appleby, Neil A. Salmon, David A. Wikner, Proceedings of SPIE Vol. 5410, (2004)

[2] Salmon, N.A. "Scene simulation for passive and active millimetre and sub-millimetre wave imaging for security scanning and medical applications", SPIE Passive Millimetre-Wave and Terahertz Imaging and Technology, London, October, (2004)

[3] Salmon, N.A. et al, "Interferometric aperture synthesis for next generation passive millimetre wave imagers", SPIE Europe Security+Defence, 'Millimetre Wave and Terahertz Sensors and Technology', Edinburgh, September, (2012) 